\title{
TECHNICAL IMPLEMENTATION OF INDIVIDUAL ELEMENTS OF THE DIGITAL TWIN CONCEPT
}

\author{
Alekseev Anton, Efremov Vladislav, Lyadsky Daniil \& Potekhin Vyacheslav \\ Peter the Great St. Petersburg Polytechnic University
}
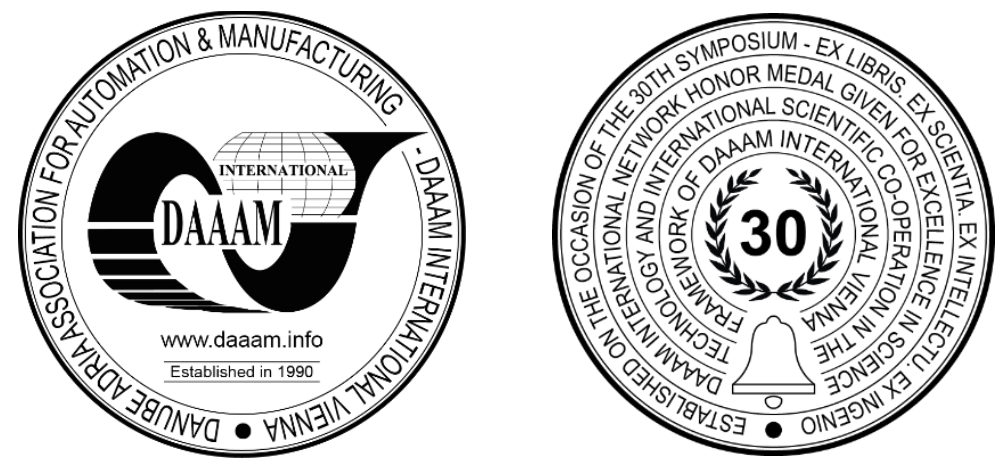

This Publication has to be referred as: Alekseev, A[nton]; Efremov, V[ladislav]; Lyadskiy, D[aniil] \& Potekhin, V[yacheslav] (2020). Technical Implementation of Individual Elements of the Digital Twin Concept, Proceedings of the 31st DAAAM International Symposium, pp.0254-0260, B. Katalinic (Ed.), Published by DAAAM International, ISBN 978-3-902734-29-7, ISSN 1726-9679, Vienna, Austria

DOI: $10.2507 / 31$ st.daaam.proceedings.035

\begin{abstract}
The article shows the relevance of using digital twins. The authors have embodied individual technical elements of the digital twin concept and demonstrated the successful results of their application. The work shows predictive analysis, technologies of the industrial Internet of things and cloud distributed control systems. The section of the industrial Internet of things demonstrates the use of a system of interconnected computer networks and connected industrial (production) facilities. This technology is used to monitor and control the condition of process equipment. The section with predictive analysis shows the possibility of using the accumulated data in the implementation of mathematical models and their use for practical purposes, for example, predicting the state of an object. The section of cloud distributed control systems shows the possibility of effectively implementing the functions of distributed control systems and the functions of operators for managing continuous processes in the cloud infrastructure and integration with the IIoT architecture. The conclusion shows the prospect of using a fully functional digital twin, against the background of the successful implementation of its individual parts.
\end{abstract}

Keywords: Industry 4.0; Digital Twin; DCS; IIoT; ML

\section{Introduction}

Modern production facilities need to get more and more operational data in order to increase production capacity and reduce costs. Today, there are MES-type data collection systems, but there are no widespread applications and practical examples of implementing Digital twins with forecasting and management capabilities. The solution to this problem in the near future should be the use of such concepts and techniques as: Industrial Internet of Things - IIoT , Cloud DCS and Digital Twin Applications based on Machine Learning models. The expected effect of implementing monitoring systems based on industrial Internet of things technologies is:

- Reducing the percentage of low-quality products;

- Identification of the main factors influencing the appearance of low-quality products;

- Increase the productivity of technological processes by optimizing energy resources and reducing the influence of the human factor on the production process; 
- Greater observability and predictability of the entire technological process when combined with big data technologies and the concept of digital twins;

- Reducing the cost of repairs by monitoring the state of technological equipment.

This article discusses the basic concepts and practices developed in the framework of the IIoT prototype project, the cloud DCS project, and the predictive Analytics project for industrial transformers. In the future, it is planned to combine the proposed technical solutions into a single digital twin architecture based on a technological installation.

\section{Cloud DCS}

The direction of virtual automated control systems and SCADA is a global innovation trend, developed by a number of major vendors of automated control systems and several global industrial concerns. In parallel, the necessary innovative technical solutions/ specifications/ standards are being developed under the auspices of international organizations. The difficulty from a modern process management system to cloud-based DCS and Web Scada is to do so in a structured way, gradually upgrading highly integrated and vendor-specific standards into a more open structure while maintaining functionality. There is a need to study the possibility of effective implementation of the functions of distributed management systems and the functions of continuous process management operators in the cloud infrastructure and integration with the IIoT architecture [1].

The following problems can be identified in the modern automated process control system architecture:

- Lack of flexibility of classic DCS when upgrading and making changes;

- Hard functionality and resource limitations of the hardware controllers of the DCS;

- Lack of unified centralized control over making changes to the management software of the DCS;

- The need for physical presence of specialists at the site for safe maintenance and changes;

- Lack of universal solutions for continuous development-testing-integration of automated process control systems based on classic DCS.

As a solution to these problems, it is proposed to create a prototype of a cloud DCS, which allows you to build a dynamically scalable management system that is integrated on a cloud platform (with connection to I/O). When switching to the cloud architecture of the automated process control system, the problems of traditional architecture are solved as follows:

- To expand the system, you need to install only I/o modules to each station, connect them to a common subnet. The controller execution environment is located in a virtual container that can be duplicated with the program already embedded in it;

- As the program density in the controller gradually increases, you can dynamically add more RAM or cores to the virtual container;

- To support a variety of network protocols, an IoT gateway is used that can convert one protocol to another.

To solve this problem, it is necessary to redesign the architecture of the automated control system. The part of the system that is more responsible for optimizing and analyzing production processes and storing big data should be moved to the cloud space, while all segments of this cloud can exchange information with each other. it is necessary to move away from the hierarchical system to graphs, where each node has a connection with another node. To connect to the cloud space - the IoT platform - you need a device that communicates with the management layer and data collection. To do this, you can use special devices - Edge controllers - which can use an open protocol to transfer data from the lower level to the cloud, as well as perform small optimization tasks, and forward commands from the cloud to the control level. A diagram of this architecture is shown in Fig. 1.

This scheme does not replace existing automated process control systems in production, but only complements it. The focus is on scaling new production sites and supporting existing ones. It is necessary to move towards the coexistence of a modern automated process control system architecture and a cloud-based one for gradual migration [2]. To confirm the possibility of effective implementation of functions of distributed management systems in the cloud, a stand was developed that allows us to study this hypothesis. The diagram of the stand is shown in Fig. 2. MasterPLC was chosen to demonstrate the functionality of a cloud-based DCS, since this candidate can most fully replicate the functionality of a real DCS.

You can connect to the cloud over the Internet using an open vpn. In this scheme, the IoT gateway acts as an MQTT broker, through which MasterPLC transmits a message to the MQTT IO device. A MasterScada program was written to control the water pump station. You can control it both from the web interface and from the HMI panel (the panel communicates with the controller via the Modbus Protocol, where the controller acts as a slave). The IO device emits the operation of pumps that are controlled by discrete signals. In the figure below, you can see the Web-Scada interface in the web browser, the HMI panel, and the IO device. The lights on the IO device correspond to the pump number. Accordingly, you can see in the image that the numbers of active pumps correspond to the numbers of enabled pumps from the Web-Scada and HMI panel. Also, using the Terraform language, templates for automatic deployment of cloud DCS images on the stand were developed. 
When you run the developed scripts, a virtual machine with the above characteristics is automatically created on the stand, images of virtual controllers and their dependencies are downloaded from the Internet and installed, the selected project is loaded into the controller, the necessary licenses are picked up, and RunTime is started.

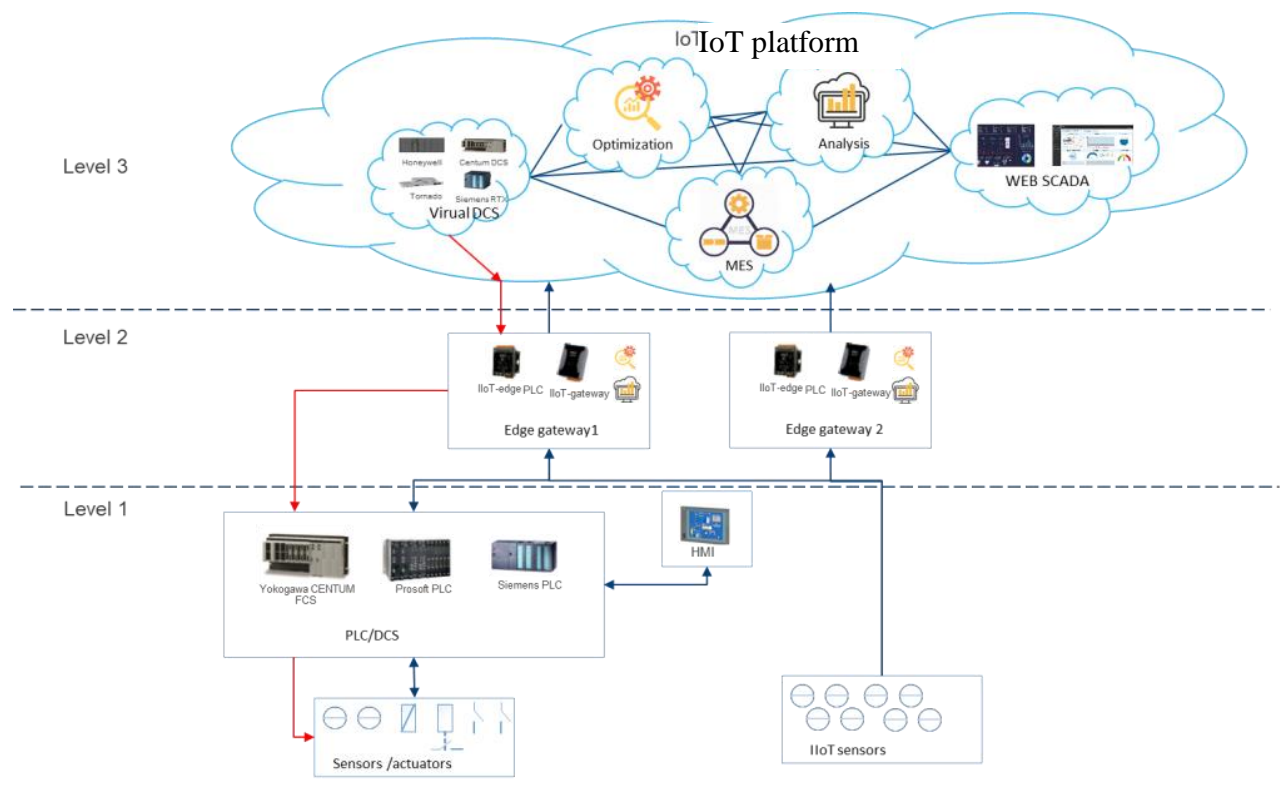

Fig. 1. IIoT architecture

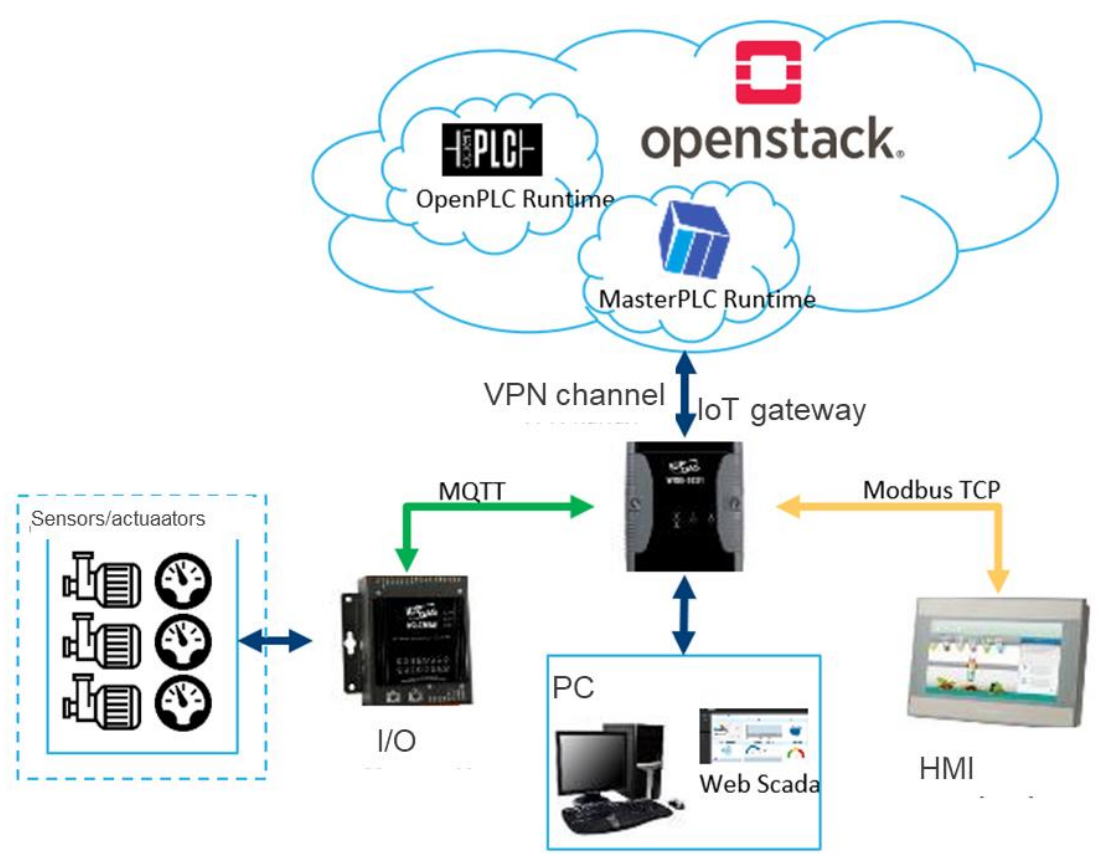

Fig. 2. IIoT stand architecture

The study made the following conclusions:

- MasterPLC technical specifications:

o Easily deploy to the cloud using deployment templates,

- Web Scada integrates with the softPLC executable program,

- Supports backup functions,

- Supports multiple communication and redundancy protocols,

o Program cycle $\sim 10 \mathrm{MS}$,

o Response delay of 130-140 MS via the Internet (ping $\sim 50 \mathrm{~ms}$ ), $\sim 10 \mathrm{~ms}$ via direct connection of devices to the IoT server. 
- MasterPLC (MasterScada) has many features of an industrial controller, is able to manage real production, where there is no need for high accuracy in the execution time of cycles;

- This virtual controller is easy to deploy in the cloud using deployment templates on the stand, since we can wrap the image in a Docker container, so after deployment it will automatically be assigned an ip address, and you can connect to the controller's web interface via a browser;

- In this way, MasterPLC can be used to implement projects with production automation, you can allocate full production management to a virtual controller, or only the part that is responsible for data analysis;

- The software supports Web Scada, which is easily integrated with the executable program, as well as its own database, where you can archive time series. Supports multiple communication and redundancy protocols;

Based on the results, the following hypothesis was confirmed: the ability to effectively implement the functions of distributed control systems and operator's workstation functions for managing continuous technological processes in the cloud infrastructure and integrating with the IIoT architecture.

\section{Industrial Internet of Things}

Modern trends and challenges in high-tech industries development arise from the development of the infrastructure and the complexity of modern industries, their territorial distribution and a variety of functional perposes, which leads to substantial problems in carrying out the control tasks [3]. The Industrial Internet of Things (IIoT) a single concept aimed to solve such problems. IIoT is a set of technologies for the implementation of an enterprise information and control system, is being formed as a unifying alternative:

- Automation technologies - AT,

- Information technology - IT,

- Operating technologies - OT.

Operational technologies that integrate operational and service personnel into production control loops, entering the IIoT perimeter, are transformed into a digital work environment, including wearable devices, human-machine interfaces and applications of systems that support decision-making.

As a result of the implementation of IIoT at the enterprise, a cross-functional, interoperable management environment is obtained, which makes it possible to increase the efficiency of solving the main problems of production management. At the same time, for all management tasks, a part of the functions remains, the implementation of which at this stage and for a specific enterprise can be effectively implemented with existing technologies without switching to IIoT technology. As the preliminary analysis shows, for systems of technical diagnostics and reliability management the range of applicability of IIoT technologies is one of the most significant.

This is determined by the following factors:

- For high-quality diagnostics and continuous monitoring of the equipment condition, in addition to primary sensors as part of the process control system, it is often necessary to install additional sensors, the integration of which into the process control system will be ineffective both in terms of the cost of adding one channel and in terms of its further maintenance;

- Building models for predicting the technical state, calculating the residual life, analyzing the causes of failures, etc. requires the accumulation and processing of large arrays of continuous data, which is not efficient to solve both by means of the process control system and standard MES tools, since the functions of these systems are characterized by other discretization values, storage depths and data volumes;

- The information required for diagnostics and reliability management is formed both at the level of the process control system and at the MES level;

- In the tasks of technical diagnostics and reliability management, a significant amount of operations are performed by personnel whose activities are poorly automated and poorly integrated into information systems.

Despite all the talk about the Fourth Industrial Revolution, some companies still acquire and use industrial equipment) without using IIoT technology to connect it to IT systems. The problem is that when companies launch projects that are designed to connect industrial OT equipment to IT systems, those projects often find themselves at a standstill, as evidenced by a Cisco survey that found three-quarters of IoT projects fail. Either during the IIoT Application Concept (POC) process, or later when these companies are trying to take their IIoT applications out of the lab into the field. Companies often experience difficulties that prevent them from developing or deploying an application. One of the main challenges leading to the failure of these projects is the complexity of building the IIoT infrastructure needed to bridge the gap that separates OT from IT [4].

With mobile and web apps, companies can leverage common coding skills to create an app in the cloud and then deliver it to users on their already connected smartphones and laptops. To develop and deploy IIoT applications, companies must build their own bridge between IT systems and OT equipment endpoints. 
To do this, they need:

- Use various communication protocols to integrate embedded modules, gateways and other peripheral devices into your industrial equipment;

- Connect these peripherals to wireless networks and then manage these connections to minimize data transfer costs and, for battery-powered peripherals, energy use;

- Create APIs that integrate peripheral data into cloud IT systems;

- Ensuring data protection by implementing a cybersecurity policy and data encryption (both server-side and client-side encryption, as well as transport encryption) [5].

\section{Predictive analysis}

In connection with the improvement of quality and technological re-equipment of industrial and energy enterprises, many of them have installed systems for monitoring the condition of objects, whether it be a machine tool or a transformer, which daily record indicators of many parameters. This leads to the fact that information storage systems are filled with a huge amount of unclaimed data. Naturally, many businesses want to use this information to their advantage. It is important to note that complex technological objects are always individual, even if they are objects of the same type. Thus, the resulting models demonstrate an advantage over classical diagnostic methods in that they can adjust to the behavior of the object in each individual case.

One example of the use of accumulated statistics is the development of predictive models. This is a rather demanded task for a number of reasons; firstly, a well-designed model is able to predict the wear period of the model, which will allow you to more accurately plan the time of maintenance, avoiding unnecessary or unplanned equipment downtime. Secondly, the absence of the need for knowledge of the subject area of the simulated object will reduce the degree of qualification of working personnel or the cost of calling specialists.
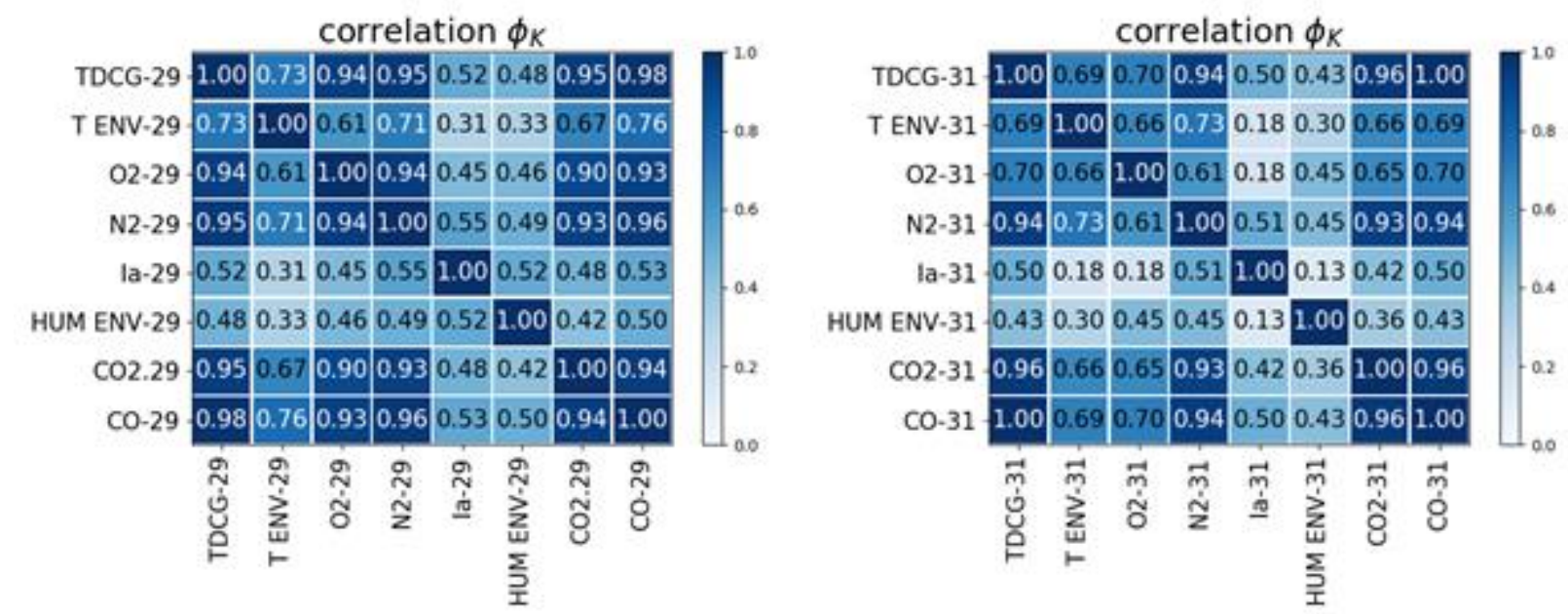

Fig. 3. Calculation of nonlinear correlation coefficients for the 29th and 31st transformers, respectively
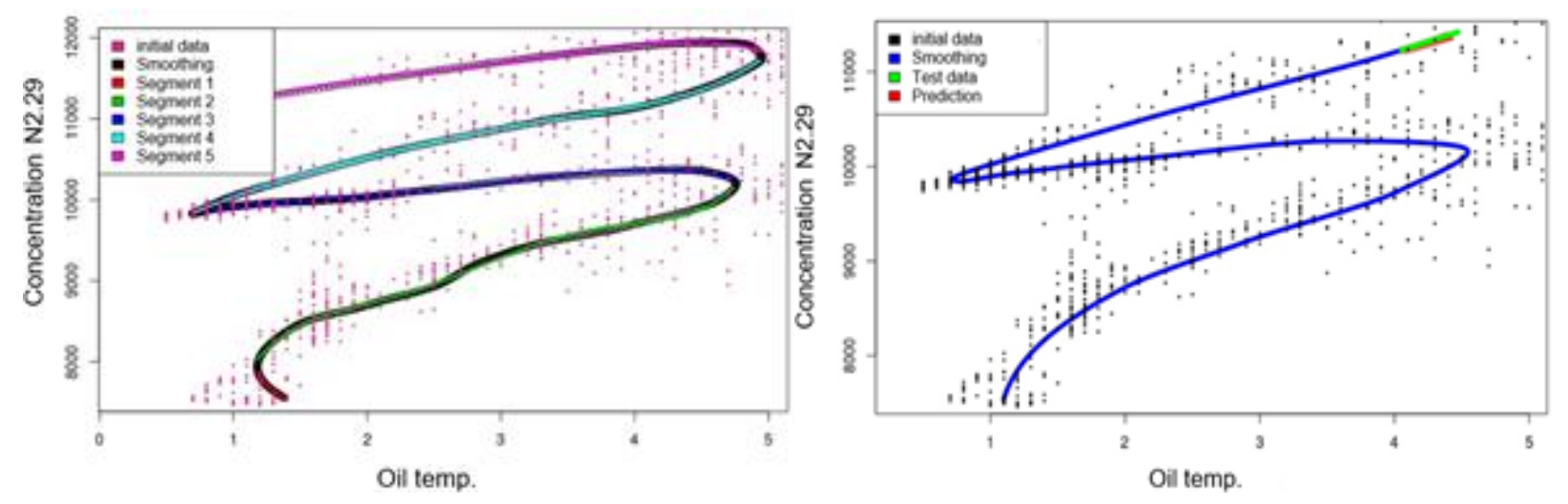

Fig. 4. Segmentation and forecast N2 respectively 
The development of two weekly predictive models of the level of concentrations of oxygen-containing gases was carried out on the basis of the transmitted statistics of 39 oil transformers. To solve this problem, modern methods of data analysis and machine learning were applied.

First of all, transformers with more complete accumulated statistics were selected from the data array and data from two monitoring systems responsible for oil and electrical parameters were compared. These were the 29th and 31st transformers. Further, the processing of raw data was done by standardizing the data and removing outliers. By analyzing the data [6], the dependence of the predicted parameters (gas concentration) on technological parameters (temperature, humidity, load currents) was found and the main predictive factor was obtained. Next, a prediction algorithm based on piecewise linear functions was proposed.

It can be seen that the model obtained on one transformer can be transferred to the prediction of another transformer. Thus, we can say that the proposed algorithm works successfully. In addition, it has also been successfully used to predict other gases $(\mathrm{CO}, \mathrm{CO} 2)$. The advantages and disadvantages of the technique are described below.

\section{Advantages:}

- Method results are easy to interpret,

- Simple and straightforward to learn,

- Universal, the model can be applied to a group of similar devices.

\section{Disadvantages:}

- It did not work out completely to "get away" from the time series. Indirectly through the temperature forecast, it is present in the concentration forecast. In theory, you can use a weather forecast;

- The concentration of gases depends not only on temperature, but also on other gases, humidity, network load (weak). Using this technique, the influence of these parameters is not considered, which can greatly affect the forecast accuracy.

As an attempt to compensate for these shortcomings, the neural network method was used. As a result, methodologies based on machine learning methods were developed and applied to predict gas concentrations in 39 oil transformers, as an analogue of classical methods of analysis of dissolved gases [7]. This approach allows you to create models (digital twins) of production facilities, in this case, oil transformers, which fully automates the monitoring of the state of the object, and also builds predictive models of the state of the object for some time in advance. This approach will reduce the requirements for the qualifications of employees, as well as organize scheduled maintenance work on the facility. Models generated by these methods can be deployed to cloud DCSs, following the architecture proposed in [8].

\section{Conclusion}

The problem of modern cyber-physical control systems is the ineffective use and processing of the information received. DCS are not flexible and easily scalable when fully modernized production. The first problem is solved by using IIoT technologies for data collection and machine learning technologies for data processing. The second problem is solved by applying the cloud DCS described in this article. As a result of the study and the implementation of a number of practical tasks, the following were developed: the target architecture of IIoT systems, a predictive model for data analysis, a prototype of a cloud DCS. The results are fully consistent with the tasks.

The positive results of the implementation of the proposed ideas will be:

1. reduction of expenses for production modernization;

2. accumulation and processing of knowledge about industrial facilities;

3. reducing equipment repair costs.

On the other hand, the implementation of these ideas will be labor intensive in the initial stages, since the migration of existing control systems will be required. In the future, it is planned to combine the proposed technical solutions into a single digital twin architecture based on a technological installation.

\section{Acknowledgments}

The paper is published in the framework of the project Erasmus+ 573545-EPP-1-2016- 1-DE-EPPKA2-CBHE-JP and describes the part of the project conducted by Peter the Great St. Petersburg Polytechnic University and also with the support of a programme World Level Research Centre "Advanced Digital Technologies" in the direction of research "Promising Platform Solutions for the Integration of Industrial Technologies of Cyber-Physical and Artificial Intelligence Systems." 


\section{References}

[1] Alekseev A.P., Efremov V.V., Potekhin V.V., Zhao Y., Du H. (2020) Digital Twin Analytic Predictive Applications in Cyber-Physical Systems. In: Arseniev D., Overmeyer L., Kälviäinen H., Katalinić B. (eds) Cyber-Physical Systems and Control. CPS\&C 2019. Lecture Notes in Networks and Systems, vol 95. Springer, Cham. - P. 1-2

[2] Colombo, Armando \& Karnouskos, Stamatis \& Bangemann, Thomas. (2014). Towards the Next Generation of Industrial Cyber-Physical Systems. 10.1007/978-3-319-05624-1_1. - P. 12

[3] Branko Katalinic, Dmitry Kostenko, Vadim A. Onufriev, Vyacheslav Potekhin (2019). Cyber-Physical systems in complex technologies and process control. (eds) Cyber-Physical Systems and Control. CPS\&C 2019. Lecture Notes in Networks and Systems, vol 95. Springer, Cham. - P. 40-54

[4] http://proxy.library.spbu.ru:2242/document/8026132/?reload=true, Ohyun, J. Internet of Things for Smart Railway: Feasibility and Application / J. Ohyun // IEEE Internet of Things Journal. , Accessed on: 2020-03-14.

[5] Gastermann, B., Stopper, M., Kossik, A., Katalinic, B. (2015). Secure implementation of an on-premises cloud storage service for small and medium-sized enterprises. In: Proceedings of DAAAM International Symposium on Intelligent Manufacturing and Automation, DAAAM 2014, Vienna, Austria, vol. 100, no. C, pp. 574-583

[6] https://www.rosseti.ru/investment/standart/corp_standart/doc/CTO_34.01-23-003-2019.pdf, CTO 34.01-23-0032019, Accessed on: 2020-03-28.

[7] M. Baaka, R. Koopmana, H. Snoekb, S. Klous. A new correlation coefficient between categorical, ordinal and interval variables with Pearson characteristics / arXiv — 2019. - P. 3-30

[8] Kostenko D., Kudryashov N.; Maystrishin M., Onufriev V., Potekhin V., Vasiliev A. (2018) Digital twin applications: diagnostics, optimisation and prediction, In Katalinic B. (eds) Proceedings of the 29th DAAAM International Symposium, Vienna, Austria — P. 2-3 